\title{
Carcinossarcoma Uterino: Relato de um Caso com Invasão da Bexiga, Mimetizando Mullerianose com Transformação Maligna
} Carcinosarcoma of the Uterus: Report of a Case with Invasion of the Urinary Bladder, Resembling Mullerianosis with Malignant Transformation Carcinosarcoma Uterino: Reporte de un Caso de Invasión de la Vejiga, Mimetismo Mülleriano con Transformación Maligna

\author{
Daniel Cury Ogata ${ }^{1}$, Wilson S. F. Busato ${ }^{2}$, Gilberto Laurindo Almeida ${ }^{3}$, Elisiário Pereira Neto ${ }^{4}$, Stefan Gerlach Braz Moreira ${ }^{5}$, \\ João Vitor Quadra Vieira dos Santos 5
}

\section{Resumo}

Introduçáo: Os carcinossarcomas uterinos são neoplasias infrequentes, correspondendo a apenas 3-9\% de todas as neoplasias ginecológicas, representam $48 \%$ de todos os sarcomas, e possuem incidência de 8,2 por 1 milhão mullheres/ano. As principais manifestaçóes clínicas são o sangramento vaginal anormal em idade pós-menopausa e dor abdominal. Caracterizam-se morfologicamente por elementos epiteliais e estromais. Podem eventualmente invadir estruturas adjacentes, inclusive a bexiga urinária. Relato do caso: Apresentamos um caso de carcinossarcoma uterino com invasão de bexiga mimetizando mullerianose com transformação maligna. A paciente do sexo feminino de 69 anos apresentava hematuria macroscópica como queixa única. Durante a investigação diagnóstica, após cistoscopia com exame anatomopatológico, foi sugerida a hipótese de mullerianose com transformaçáo benigna. A paciente foi então encaminhada para ressecção cirúrgica da neoplasia. Durante o procedimento cirúrgico, notou-se a existência de uma massa tumoral uterina que invadia a bexiga urinária, e, após análise anatomopatológica, mostrou tratar-se de um carcinossarcoma uterino com invasão vesical. Conclusão: Manifestaçôes incomuns em doenças com grande potencial de malignidade, além de retardar o processo diagnóstico, interferem diretamente no prognóstico do paciente.

Palavras-chave: Neoplasias Uterinas; Carcinossarcoma; Útero; Bexiga Urinária

Trabalho realizado no Laboratório PHD e Universidade do Vale do Itajaí (UNIVALI). Itajaí (SC), Brasil.

${ }^{1}$ Médico Patologista. Mestre em Cirurgia pela PUC-PR. Docente do Curso de Medicina da UNIVALI.

${ }^{2}$ Médico Urologista do Hospital e Maternidade Marieta Konder Bornhausen. Doutor em Clínica Cirúrgica pela UFPR. Docente do curso de Medicina da UNIVALI.

${ }^{3}$ Médico Urologista do Hospital e Maternidade Marieta Konder Bornhausen.

${ }^{4}$ Médico Patologista, Docente do Curso de Medicina da UNIVALI.

${ }^{5}$ Acadêmicos de Medicina da UNIVALI.

Endereço para correspondência: Daniel Cury Ogata.Rua Expedicionário Marquetti, 65. Itajaí (SC), Brasil. CEP 88301-620. E-mail: daniel.ogata@gmail.com. 


\section{INTRODUÇÃO}

Os carcinossarcomas uterinos, também conhecidos como tumores mullerianos mistos malignos, são neoplasias raras, altamente agressivas e com prognóstico sombrio. Acometem geralmente pacientes idosas e frequentemente apresentam-se em estágio avançado no momento do diagnóstico ${ }^{1}$.

A mullerianose da bexiga urinária é um mimetizador tumoral muito raro, sendo definido como lesóes com, pelo menos, dois epitélios do tipo mulleriano, tais como: endometrial (endometriose), endocervical (endocervicose) e tubáreo (endossalpingiose) ${ }^{2}$. Entre estes, o mais comum é a endometriose, que na bexiga urinária perfaz aproximadamente $1 \%$. Nestes casos, a transformação maligna é extremamente rara, com apenas seis casos relatados. Destes, cinco eram adenocarcinomas endometrioides e um adenossarcoma ${ }^{3}$.

Relatamos um caso de paciente com lesão polipoide vesical, cuja análise histológica mostrou tratar-se de carcinoma seroso infiltrando tecido com padrão endometrial. Posteriormente, a cirurgia radical revelou que, na verdade, a lesão era de origem uterina e polipoide com invasão vesical, mimetizando transformação maligna em foco de mullerianose.

\section{DESCRIÇÃO DO CASO}

Paciente de 69 anos procurou auxílio médico devido à queixa de hematúria macroscópica. Notou o surgimento desse sintoma há aproximadamente 3 meses. Nenhuma outra queixa referente aos outros sistemas foi significativa. Ao exame físico, foi constatado que a paciente pesava $89 \mathrm{Kg}$ e media $162 \mathrm{~cm}$ de altura, atingindo um índice de massa corporal (IMC) de $33,62 \mathrm{Kg} / \mathrm{m}^{2}$. O abdômen era flácido e indolor, sem sinais de ascite ou adenomegalias evidentes. $\mathrm{O}$ exame especular mostrou prolapso uterino grau I e o toque retal náo acrescentou nenhum dado significativo. Relatou não fazer terapia de reposição hormonal. Uma cistoscopia realizada mostrou uma lesão polipoide, inserida na parede posterior da bexiga, imediatamente abaixo do trígono vesical. A lesão foi excisada e o exame histopatológico revelou um carcinoma seroso infiltrando tecido do tipo endometrial, sugerindo transformação maligna em restos mullerianos (mullerianose).

Aproximadamente dois meses após o procedimento inicial, a paciente foi submetida à nova cistoscopia com biópsia do leito cirúrgico. Essa nova biópsia mostrou quadro de cistite glandular cística com foco contendo tecido endometrial, mas sem evidências de neoplasia residual. A paciente foi mantida em observação. Foi quando em curto espaço de tempo, após a segunda cistoscopia, cerca de seis meses, a paciente reapareceu com a hematúria macroscópica. Realizou-se nova cistoscopia que revelou recidiva da lesão. A tomografia axial computadorizada (TAC) contrastada, realizada logo após, mostrou falha de enchimento, caracterizando a massa tumoral (Figura 1). Essa massa tumoral predominava na bexiga, porém tinha a mesma densidade do tecido uterino. Uma exanteração pélvica anterior com esvaziamento ganglionar pélvico bilateral foi recomendada como abordagem cirúrgica. O exame macroscópico da peça cirúrgica mostrou lesão polipoide inserida em fundo uterino com perfuração e transfixação da parede uterina, invadindo a bexiga (Figura 2). A microscopia mostrou neoplasia bifásica, constituída por elemento epitelial, caracterizado pelo carcinoma seroso (Figura 3) e componentes estromais, representados pelo condrossarcoma e rabdomiossarcoma (Figura 4). O diagnóstico final foi de carcinossarcoma com elementos heterólogos, invadindo a bexiga. Os linfonodos encontravam-se livres de comprometimento neoplásico. Atualmente a paciente encontra-se em tratamento quimioterápico adjuvante e, cinco meses após a cirurgia, não apresenta sinais de recidiva.

\section{DISCUSSÃO}

Os sarcomas uterinos são tumores altamente agressivos e raros, representando cerca de 3-9\% de todas neoplasias malignas ginecológicas. Os principais tipos incluem o carcinossarcoma, leiomiossarcoma, sarcoma do estroma endometrial e sarcoma indiferenciado ${ }^{4}$.

Os carcinossarcomas, também chamados de tumores mullerianos mistos malignos, são originários do mesoderma mulleriano e contêm elementos tanto epiteliais como estromais ${ }^{1}$. Essas neoplasias podem ser subdivididas conforme o componente estromal em homólogo e heterólogo. Os carcinossarcomas com elementos homólogos possuem estroma maligno nativo

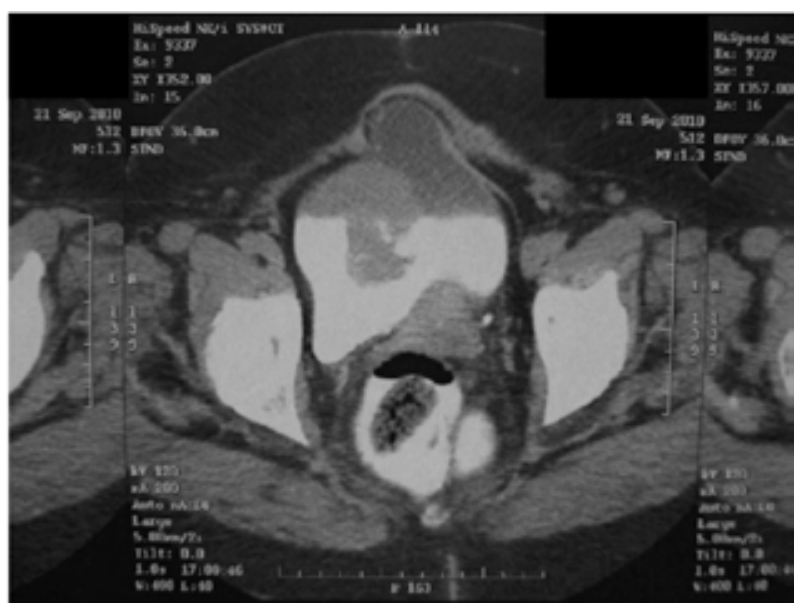

Figura 1. Tomografia axial computadorizada pélvica. A imagem mostra corte axial da pelve. Percebe-se uma falha de enchimento no interior da bexiga, representando a massa tumoral, com a mesma densidade do tecido uterino 


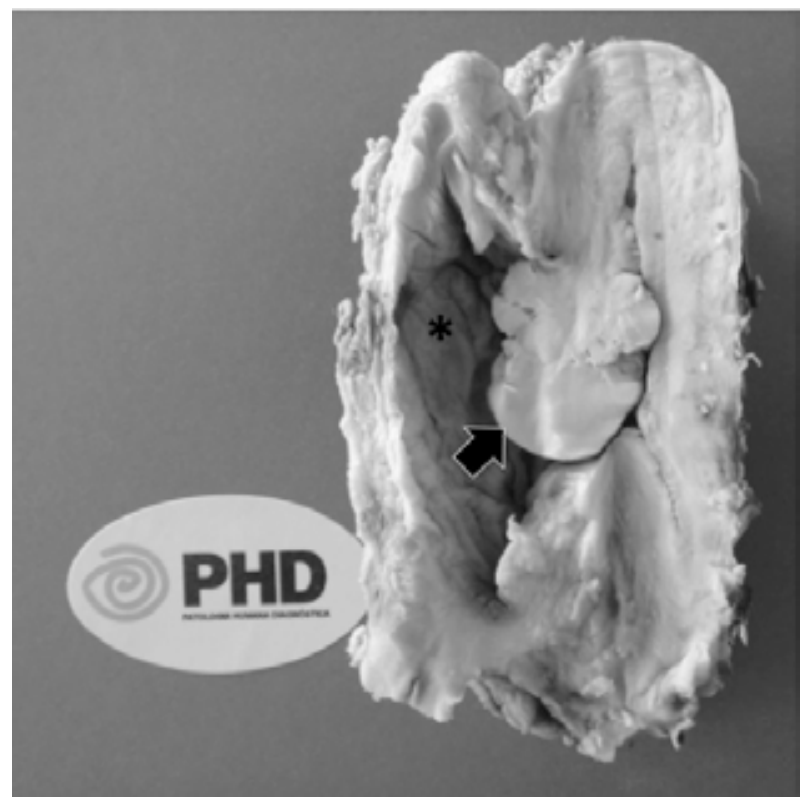

Figura 2. Espécime macroscópico mostrando tumor (seta), invadindo bexiga (asterisco)

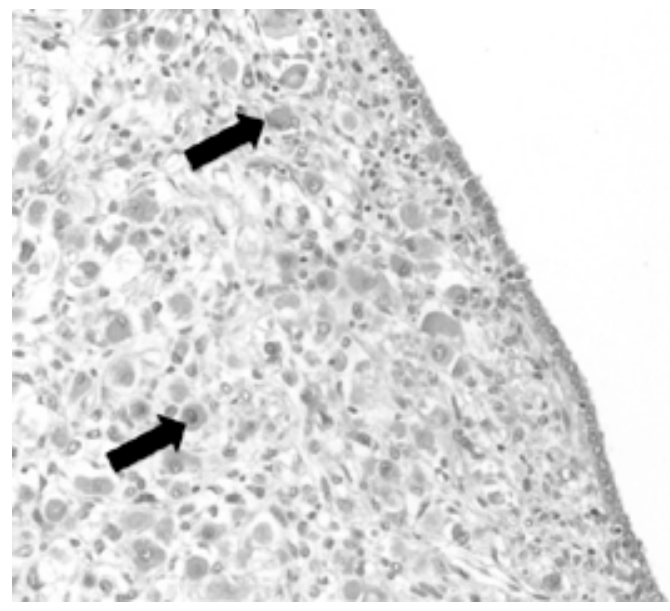

Figura 3. Exame microscópico mostrando elementos estromais heterólogos, representados por rabdomioblastos (setas) (400x HE)

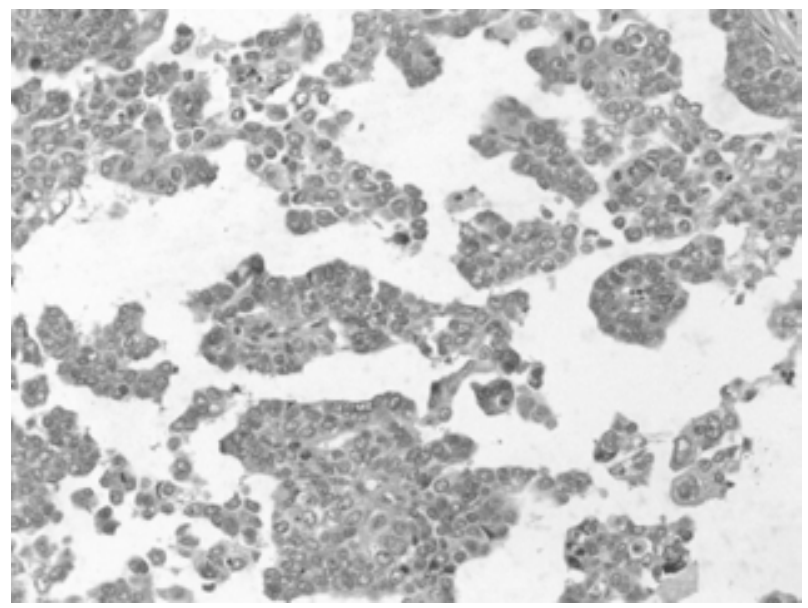

Figura 4. Exame microscópico mostrando carcinoma seroso (400x HE) do próprio útero. Já o subtipo heterólogo possui elementos sarcomatosos observados em sítios extrauterinos, tais como: tecido muscular estriado, osso e cartilagem ${ }^{1}$.

Os carcinossarcomas são mais observados em mulheres climatéricas ${ }^{1}$, com média de idade no momento do diagnóstico de 58 anos $^{5}$. Esses tumores dividem muitos fatores de risco com os adenocarcinomas endometriais, entre eles: obesidade, nuliparidade e suplementação com estrogênios. A irradiação pélvica prévia e o uso de tamoxifeno também têm sido relacionados como fatores envolvidos nestas neoplasias. Enquanto o uso de contraceptivo oral é considerado como fator de proteção dessa doença ${ }^{1}$.

Os principais sintomas dessas neoplasias são: dor e sangramento vaginal ${ }^{5}$. A hematúria macroscópica, presente neste relato, é extremamente infrequente e acabou causando confusão, uma vez que o foco da lesão passou a ser a bexiga urinária. Neste cenário, somado à imagem observada na cistoscopia e ao exame anatomopatológico, conjecturou-se a possibilidade de transformação maligna em área de mullerianose. Essa entidade é definida como complexo morfológico, composto de vários tipos de lesôes do tipo mulleriano, entre elas a endometriose. Alguns autores sugerem que essas lesóes são de origem metaplásica ${ }^{2}$. Considerando o trato genitourinário, a bexiga urinária é o sítio mais comumente envolvido pela endometriose ${ }^{3,6}$. Aproximadamente $50 \%$ das pacientes têm antecedentes de cirurgia pélvica e $12 \%$ não têm evidências dessa doença em outra topografia. A média de idade no momento do diagnóstico é de aproximadamente 35 anos. Raramente, a endometriose acomete mulheres na pós-menopausa que utilizam estrogênio ${ }^{6}$. A mullerianose já é um fenômeno raro, com pouco menos de $20 \operatorname{casos}^{7}$ relatados, e a malignizaçáo, como complicação, é mais infrequente ainda, sendo descritos apenas seis casos. Destes, nenhum tinha como componente epitelial o carcinoma seroso, presente em nosso caso ${ }^{3}$. Alguns critérios foram propostos para estabelecer a endometriose extragonodal como precursora de adenocarcinomas, sendo eles: (i) evidências claras de endometriose nas proximidades do tumor; (ii) nenhum outro tumor primário pode ser encontrado; (iii) a aparência histológica deve permitir que a origem do adenocarcinoma tenha acontecido a partir da endometriose ${ }^{3}$. Ainda assim, a mullerianose pode continuar a causar confusão diagnóstica com neoplasia vesical, transformação maligna em endometriose e envolvimento secundário de tumor extravesical ${ }^{3}$.

Infelizmente, a TAC, realizada previamente ao ato cirúrgico, não permitiu confirmar a origem uterina da lesão em nossa paciente, uma vez que ela predominava na bexiga urinária. A hematúria macroscópica como única queixa levantou a possibilidade de doença vesical. Aliás, esse achado, juntamente com sintomas irritativos, está 
entre as manifestaçôes frequentemente referidas pelas pacientes portadoras de mullerianose ${ }^{8}$. Apesar disso, a idade da paciente, a ausência de suplementaçáo com estrogênios e a raridade do diagnóstico cogitado deveriam ter motivado uma maior abrangência investigativa, incluindo o trato genital feminino, especialmente o útero. Geralmente, os carcinossarcomas dessa topografia crescem como massa sólida e vegetante com necrose e hemorragia, frequentemente inserida no fundo uterino, como observado em nosso caso. $\mathrm{O}$ miométrio pode ser invadido em vários níveis, inclusive podendo haver extensão extrauterina? .

O tratamento para os carcinossarcomas uterinos é inicialmente cirúrgico, sendo indicada a histerectomia total com amostragem linfonodal pélvica, podendo ou não ser realizada salpingo-oforectomia bilateral. $\mathrm{O}$ uso de terapias adjuvantes com radio e quimioterapia é altamente variável ${ }^{4}$, sendo recomendado por alguns autores até mesmo em tumores menos avançados, devido à alta recorrência ${ }^{1}$. Nos casos avançados, o uso de terapia adjuvante é compulsório ${ }^{1}$. Apesar disso, o prognóstico continua ruim, com média de sobrevida em cinco anos variando de 17,5 a $53 \%{ }^{4}$.

\section{CONCLUSÃO}

Em suma, os carcinossarcomas uterinos são tumores incomuns, altamente agressivos e com prognóstico sombrio. Nosso caso, apresentou manifestação clínica infrequente, causando confusão com relação ao seu sítio. O tratamento desses tumores consiste na exérese do tumor e órgãos envolvidos, além da terapia adjuvante pertinente. Devemos também salientar sobre a importância da abordagem multidisciplinar entre os cirurgióes, oncologistas e radioterapeutas, sendo de grande valor a colaboração do patologista, descrevendo a histologia particular de cada tumor, bem como definindo a sua origem.

\section{CONTRIBUIÇÕES}

Todos os autores contribuíram efetivamente na concepção e planejamento do estudo; na obtenção, análise e interpretação dos dados; e na redação e revisão crítica.

\section{Declaraçáo de Conflito de Interesses: Nada a Declarar. REFERÊNCIAS}

1. Tovar JR, Callejas MER, Bermejo JIA, Armas LFC, Martínez FGP, Navarro LC. Malignant mixed mullerian tumors. Clin Transl Oncol. 2006;8(2):29-32.

2. Koren J, Mensikova J, Mukensnabl P, Zamecnik M. Mullerionosis of the urinary bladder: report of a case with suggested metaplastic origin. Virchows Arch. 2006;449:268-71.

3. Garavan F, Grainger R, Jeffers M. Endometrioid carcinoma of the urinary bladder complicating vesical Mullerionosis: a case report and review of the literature. Virchows Arch. 2004;444:587-9.

4. Koivisto-Korander R, Butzow R, Koivisto AM, Leminen A. Immunohistochemical studies on uterine carcinosarcoma, leiomyosarcoma, and endometrial stromal sarcoma: expression and prognostic importance of ten different markers. Tumour Biol. 2011;32(3):451-9.

5. Soumarová R, Horová H, Seneklová Z, Ruzicková J, Horová I, Budiková $\mathrm{M}$, et al. Treatment of uterine sarcoma: a survey of 49 patients. Arch Gynecol Obstet. 2002;266:92-5.

6. Young RH. Non-neoplastic disorders of the urinary bladder. In: Bostwick DG, Cheng L. Urologic surgical pathology. 2nd ed. [St. Louis (Mo)]: Elsevier; 2008.

7. Guan H, Rosenthal DL, Erozan YS. Mullerianosis of the urinary bladder: report of a case with diagnosis suggested in urine cytology and review of literature. Diagn Cytopathol. 2011 Apr 28; [Epub ahead of print]. doi:10.1002/dc.21693.

8. Cruz Guerra NA, Gómez Raposo MD, Baizán García MJ, Prieto Nogal SB, Juan AG, Sierra MP. [Mullerianosis of the urinary bladder: a rare entity]. Arch Esp Urol. 2009;62(2):150-2. Spanish.

9. Rha SE, Byun JY, Jung SE, Lee SL, Cho SM, Hwang SS, et al. CT and MRI of uterine sarcomas and their mimickers. AJR Am J Roentgenol. 2003;181(5):1369-74. 


\section{Abstract}

Introduction: Uterine carcinosarcoma are infrequent neoplasms, corresponding to 3-9\% of all gynecologic neoplasms only. They represent $48 \%$ of all sarcomas, with an incidence of 8.2 per 1 million women/year. The main clinical manifestations are postmenopausal vaginal bleeding and abdominal pain. Tumors are morphologically characterized by epithelial and stromal elements. They can eventually invade adjacent structures, including the urinary bladder. Case Report: We present a case of uterine carcinosarcoma with invasion of the urinary bladder resembling mullerianosis with malignant transformation. A 69 year-old female patient had macroscopic hematuria as the single complaint. During diagnostic investigations, after cystoscopy with anatomical and pathological exam was performed, the hypothesis of mullerianosis with benign transformation was suggested. The patient was thus submitted to surgical resection of this lesion. During the surgical procedure, we noticed the existence of a uterine tumor mass that was invading the urinary bladder, and after anatomical and pathological analysis, it was identified as a carcinosarcoma of the uterus with invasion of the urinary bladder. Conclusion: Unusual manifestation of diseases with great malignant potential, in addition to delay the process of diagnosis, directly interferes with the patient's prognosis.

Key words: Uterine Neoplasms; Carcinosarcoma; Uterus; Urinary Bladder

\section{Resumen}

Introducción: Los carcinosarcomas uterinos son neoplasias poco frecuentes, correspondiente a solo 3-9\% de todos los cánceres ginecológicos, lo que representa 48\% do todos los sarcomas, e incidencia de 8,2 por 1 millón de mujeres/ año. Las principales manifestaciones clínicas son la sangría vaginal anormal en edad post-menopausa y dolor abdominal. Se caracterizan morfológicamente por elementos epiteliales y estromales. Pueden casualmente invadir estructuras adyacentes, incluso la vejiga urinaria. Informe de caso: Presentamos un caso de carcinosarcoma uterino con invasión de la vejiga, mimetismo mülleriano con transformación maligna. La paciente de 69 años presentaba hematuria macroscópica como queja única. Durante la investigación de diagnóstico, tras cistoscopia con examen anatómico e patológico, se planteó la posibilidad de mimetismo mülleriano con transformación benigna. La paciente fue luego remitida para la resección quirúrgica del tumor. Durante el procedimiento, se observó la existencia de un tumor uterino que invadía la vejiga urinaria, y después de un análisis anatómico y patológico demostró que se trataba de un carcinosarcoma uterino con invasión de la vejiga. Conclusión: Manifestaciones inusuales de enfermedades con alto potencial de malignidad, además de retrasar el proceso de diagnóstico, interfieren directamente en el pronóstico del paciente.

Palabras clave: Neoplasias Uterinas; Carcinosarcoma; Útero; Vejiga Urinaria 